\title{
Novel Applications of Functionally Graded Nano, Optoelectronic and Thermoelectric Materials
}

\author{
Isha Bharti, Nishu Gupta, and K. M. Gupta, Member, IACSIT
}

\begin{abstract}
Functionally graded materials are a novel class of materials having unique characteristics. They are graded property materials and are used as medical implants, for thermal protection of space vehicles, as thermoelectric converter for energy conservation etc. Due to their versatility of behaviour, they are now used as nano, optoelectronic and thermoelectric materials also. Future applications demand materials having extraordinary mechanical, electronic and thermal properties which can sustain different environment conditions and are easily available at reasonable prices. The carbon nanotubes (CNT) reinforced functionally graded composite materials (FGCM) is expected to be the new generation material having a wide range of unexplored potential applications in various technological areas such as aerospace, defence, energy, automobile, medicine, structural and chemical industry. They can be used as gas adsorbents, templates, actuators, catalyst supports, probes, chemical sensors, nanopipes, nano-reactors etc.

This paper aims at elaborating the development of such materials by compiling the ongoing researches. In this regard, the research developments of newer materials by different investigators have been presented here. Brief details of the development of CNT reinforced Functionally Graded composite materials, FGM in optoelectronic applications, FG thermoelectric materials have been presented here. In these elaborations, it is shown by the respective investigators that these FGMs can be effectively used as MRI scanner cryogenic tubes, tools and dies, solar panels, photodetectors, high temperature joining materials and various structural elements etc.
\end{abstract}

Index Terms-CNT reinforced functionally graded composite materials (FGCMs), FGM in optoelectronics, FG thermoelectric materials.

\section{INTRODUCTION TO FUNCTIONALLY GRADED MATERIALS (FGMS)}

A functionally graded material (FGM) is a twocomponent composite characterised by a compositional gradient from one component to other. In contrast to traditional composites that are homogeneous mixtures and involve a compromise between the desirable properties of component materials; the significant proportions of FGMs contain the pure form of each component, hence the need for compromise is eliminated. Thus the properties of both

Manuscript received January 4, 2013; revised March 4, 2013.

Isha Bharti is with the Department of Applied Physics, Delhi Technological University, Delhi (e-mail: dtu.isha@gmail.com).

Nishu Gupta, was with the Department of Electronics and Communication Engineering, Motilal Nehru National Institute of Technology, Allahabad (e-mail: dce.nishu@gmail.com).

K. M. Gupta is with the Department of Applied Mechanics, Motilal Nehru National Institute of Technology, Allahabad (e-mail: kmgupta@mnnit.ac.in). components can be fully utilised. For example, the toughness of a metal can be mated with the refractoriness of a ceramic without any compromise in the toughness of the metal or the refractoriness of the ceramic.

Functionally graded materials may be characterized by the variation in their composition and structure gradually over the volume, resulting in corresponding changes in their properties. The materials can be designed for specific functions and applications.

Functionally graded materials (FGMs) are a new generation of engineered materials that are gaining interest in recent years. FGMs were initially designed as thermal barrier materials for aerospace structural applications and fusion reactors. FGMs also found applications in structural components operating under extremely high-temperature environment. As an example, FGMs based on ceramic reinforcement in metal matrix are able to withstand hightemperature environments due to better thermal resistance of ceramic constituents, while the metal constituents enhance their mechanical performance and reduce the possibility of catastrophic fracture. FGMs are the composite materials in which the content of reinforcement is gradually varied in some direction to achieve gradient in properties. Due to graded variation in the content of constituent materials, the properties of FGMs undergo appreciable and continuous change from one surface to another, thus eliminating interface problems and diminishing thermal stress concentrations. There are many areas of application for FGMs. The concept is to make a composite material by varying the microstructure from one material to another material with a specific gradient. This enables the material to have the best of both materials. If it is for thermal or corrosive resistance, or malleability and toughness, both strengths of material may be used to avoid corrosion, fatigue, fracture and stress corrosion cracking.

\section{A. Types of FGMs}

Depending upon the nature of gradient, the functionally graded materials (composites) may be grouped into following types [1].

1) Fraction gradient type (Fig. 1a)

2) Shape gradient type (Fig.1b)

3) Orientation gradient type (Fig.1c)

4) Size (of material) gradient type (Fig.1d)

\section{CNT REINFORCED FGM COMPOSITES [2]}

CNT reinforced metal or ceramic matrix functional graded composites exhibit improvements in properties such as thermo- mechanical, lightweight, dimensional stability, barrier properties, flame retardancy heat resistance and 
challenging technology in future. Compared to metals these show better higher strength-to- density ratios, higher stiffness-to-density ratios, better fatigue and wear resistance, better elevated temperature properties (higher strength-lower creep rate. In contrast to composites materials, FGcomposites have new unique properties like fabricate directional mechanical properties, higher temperature capability (lower thermal expansion properties), and excellent fatigue and fracture resistance. However, it is difficult to meet stringent dimensional stability requirements during gradation and results in higher cost of the product.

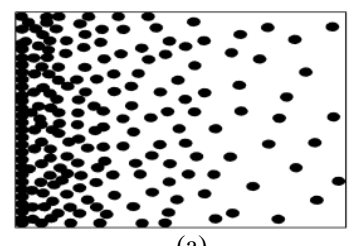

(a)

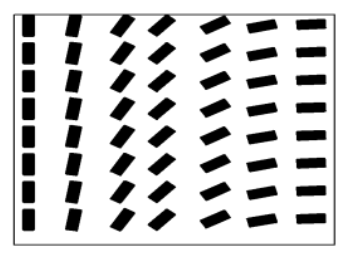

(c)

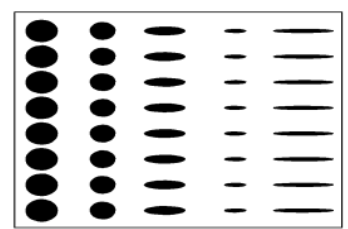

(b)

(d)

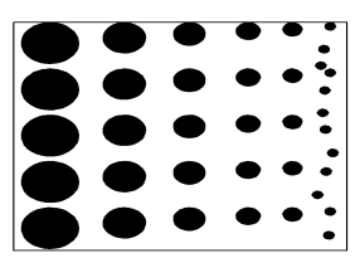

Fig. 1. Based on vividities of gradients, different types of functionally graded materials may be of (a) fraction gradient type, (b) shape gradient type,(c) orientation gradient type, and (d) size gradient type [1].

\section{A. Applications of CNT in FGM}

CNT reinforced metal matrix functional gradedcomposites due to their unique combination of hardness, toughness and strength are universally used in cutting tools, drills, machining of wear resistant materials, mining and geothermal drilling. CNT reinforced functional gradedcomposite materials have the ability to generate new features and perform new functions that are more efficient than larger structures and machines. Due to functional variation of FGM-materials, their physical/chemical properties (e.g. stability, hardness, conductivity, reactivity, optical sensitivity, melting point, etc.) can be manipulated to improve the overall properties of conventional materials. Some of the current and futuristic applications of FGM are listed as follows [2].

1) Commercial and Industrial: Pressure vessels, Fuel tanks, Cutting tool inserts, Laptop cases, Wind turbine blades, Firefighting air bottles, MRI scanner cryogenic tubes, Eyeglass frames, Musical instruments, Drilling motor shaft, X-ray tables, Helmets.

2) Automobiles: (SiC-SiC) Combustion chambers,(AI$\mathrm{SiC})$ Engine cylinder liners, (SiCw/AI-alloy) Diesel engine pistons, (E-glass/epoxy) Leaf springs, (AI-C) Drive shafts, (AI-SiC) Flywheels, Racing car brakes, (SiCp/AI-alloy) Shock absorbers.

3) Aerospace equipment and structures: (TiAl-SiC fibers) Rocket nozzle, Heat exchange panels, Spacecraft truss structure, Reflectors, Solar panels, Camera housing, Turbine wheels (operating above 40,000 rpm),Nose caps and leading edge of missiles and Space shuttle.

4) Sub-marine: (Carbon and glass fibers) Propulsion shaft, (Graphite/Epoxy) Cylindrical pressure hull, (Glass/Epoxy) Sonar domes, Composite piping system, (AI-SiC) diving cylinders.

5) Biotechnology: The functional gradient nanohydroxyapatite reinforced polyvinyl alcohol (nanoHA/PVA) gel biocomposites can be prepared through a layer-by-layer casting method combining with freeze/thaw cycles technology. NanoHA/PVA gel biocomposites have been a promising and excellent artificial articular cartilage repair material. Compared to hydrogel-based materials such as poly(vinyl alcohol) (PVA), poly(lactic acid) and chitosan, nanoHA/PVA gel biocomposites possesses superior bioactivity and mechanical properties because of the nanoHA existence in the composites. [3], [4]

\section{B. Other Applications of CNT Reinforced FGM}

Potential applications of FGM are both diverse and numerous. Some more applications of CNT in FGM having recent applications are the following [2]:

1) CNT reinforced functionally graded piezoelectric actuators.

2) As furnace liners and thermal shielding elements in microelectronics.

3) CNT reinforced functionally graded tools and dies for better thermal management, better wear resistance, reduce scrap, and improved process productivity.

4) CNT reinforced functionally graded polyestercalcium phosphate materials for bone replacement.

\section{FGM in Optoelectronic DEVices [5]}

Functionally graded materials (FGMs) are materials in which some particular physical properties are changed with dimensions. Properties of such materials can be described by material function $\mathrm{f}(x)$. In homogenous materials this function is constant, like in Fig. 2a. In case of a junction of two different materials, function $\mathrm{f}(\mathrm{x})$ has a stair-shape (Fig. 2b).
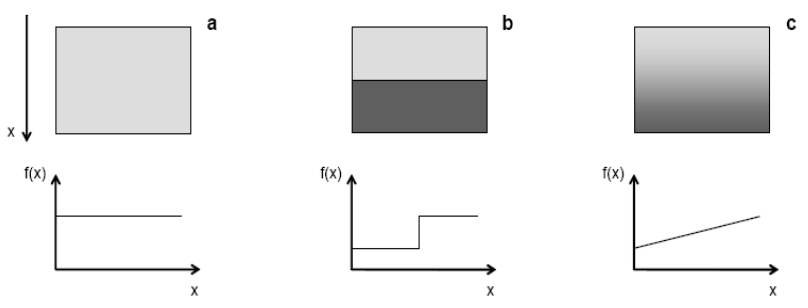

Fig. 2. Schematic representation of materials function in different structures; homogenous material (a), junction (b), FGM (c)

In FGM, this material function should be continuous or quasi-continuous. It means that the particular properties change continuously or quasi continuously along one direction, like it is shown in Fig. 2c. In many cases, FGM can be presented as a composition of several connected thin layers.

Depending on the number of directions the proprieties changed, we can discriminate as 1- dimensional, 2dimensional or 3-dimensional FGM. It can be mathematically described for 3-D FGM as 


$$
\frac{\mathrm{d} F}{\mathrm{~d} x} \neq 0, \frac{\mathrm{d} F}{\mathrm{~d} y} \neq 0, \frac{\mathrm{d} F}{\mathrm{~d} z} \neq 0
$$

where $F(x, y, z)$ is the material function.

\section{A. Possible Applications of FGM in Optoelectronics}

Now-a-days the graded materials are widely used for antireflective layers, fibers, GRIN lenses and other passive elements made from dielectrics, and also for sensors and energy applications. For example, the modulation of refractive index can be obtained in such components through the change in material composition. Another possibility is to apply concept of gradation in semiconductor active devices. In semiconductors the material function can describe energetic bandgap, refractive index, carrier concentration, carrier mobility, diffusion length, built-in electric field and another property which influence the parameters of optoelectronic devices.

\section{B. High-Efficient Photodetector and Solar Cells [5]}

The fundamental limitation of the efficiency of homogenous silicon solar cells is the constant energetic band-gap width in bulk material. Because the high-energetic radiation is absorbed in a shallow layer under surface, it is necessary to form electric field in close vicinity to the surface. Generated carriers can effectively be separated in electric field; therefore the diffusion length of carriers should be longer than junction depth.

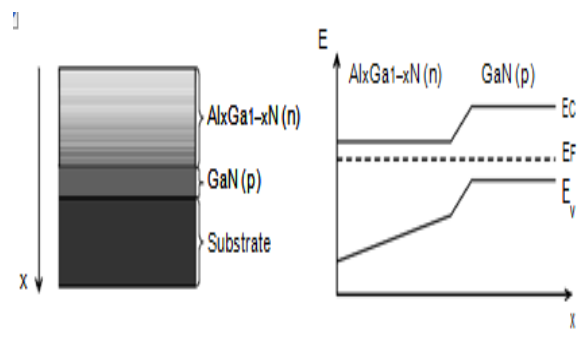

Fig. 3. Schematic structure of $p-n$ photodiode with graded layerelectric field, therefore the diffusion length of carriers should be longer than junction depth [3].

Another factor which decreases carrier generation efficiency is the difference of energetic band-gap and absorbed photons energy. By using materials with gradation of energetic band-gap, it is possible to match the absorption edge with band-gap, which improves generation efficiency. The appliance of cascade of junctions with different energetic band-gap width can be one of the solutions. Another way to overcome this limitation is the use of graded material. The idea of such device is shown in Fig. 3.

\section{FGM THERMOELECTRIC MATERIALS}

A good thermoelectric material possesses a large Seebeck coefficient, high electrical conductivity and low thermal conductivity. A high electrical conductivity is necessary to minimize the Joule heating, while a low thermal conductivity helps to retain heat at the junctions and maintain a large temperature gradient. These materials, however, have a constant ratio of electrical to thermal conductivity (Widemann-Franz-Lorenz law); so it is not possible to increase one without increasing the other. Metals best suited to thermoelectric applications therefore posses a high Seebeck coefficient. Unfortunately, most of these metals possess Seebeck coefficients in the order of 10 microvolts/K, resulting in generating efficiencies of only fractions of a percent. Therefore, the development of formulated semiconductors with Seebeck coefficients in excess of 100 microvolts $/ \mathrm{K}$ increased the interest in thermoelectricity. Earlier it was not known that the semiconductors were superior thermoelectric materials due to their higher ratio of electrical conductivity to thermal conductivity, when compared to metals.

A higher figure-of-merit $Z$ of a thermoelectric material shows a higher performance at a specific narrow temperature range [4]. On the other hand, the specific temperature can be shifted to higher temperature by increasing the carrier concentration. Bismuth telluride $\left(\mathrm{Bi}_{2} \mathrm{Te}_{3}\right)$, lead telluride $(\mathrm{PbTe})$ and $\mathrm{Si}-\mathrm{Ge}$ alloy $(\mathrm{SiGe})$ are used for low, medium and high temperature range, respectively. Usually, a monolithic and uniform thermoelectric material is used, though a temperature gradient exists in the thermoelectric material. Therefore, each part has not proper carrier concentration for each temperature. Two times of higher performance than a traditional thermoelectric material can be expected, if the proper carrier concentration gradient is performed to fit with the temperature gradient. Performing stepwise change of carrier concentration is also a performing method for practical application. That is a fundamental concept of energy converting FGM.

It is essential to choose a proper material for each part to fit the temperature gradient. The proper material is a material with proper carrier concentration and a proper compound to match the temperature of each part along the temperature gradient. FGM joining of these materials and fitting electrodes with FGM interface are also core technique, because thermal stress relaxation caused by the difference of thermal expansion coefficient is important at a high temperature.

\section{A. PbTe Based FGM Thermoelectric Material [6]}

Performing higher $Z$ over a wide temperature range is essential to obtain higher thermoelectric efficiency $\eta$. Figure 4 shows the temperature dependence of $Z$ for 5 kinds of n-type PbTe with different carrier concentration, which was induced from a report of ZT-data by Goff and Lowney as the parameter of carrier concentration. Every Z-curve has a maximum value, and the corresponding temperature shifts with the carrier concentration. If these materials are joined to fit with the temperature gradient, a higher performance than traditional thermoelectric materials can be expected.

When the FGM structure is applied to PbTe, the conversion efficiency can be improved remarkably. Five kinds of PbTe in Fig 4 are joined in sequence at the intercept temperatures of 530, 640, 750 and $815 \mathrm{~K}$. This segmented $\mathrm{PbTe}$ has five kinds of different carrier concentration and shows five maximum values of $Z$ at different temperatures.

Comparing with the ordinal monolithic $\mathrm{PbTe}$ with a carrier concentration of $3 \times 10^{25} / \mathrm{m}^{3}$, which shows the highest efficiency in monolithic PbTe, the FGM exhibits a larger $Z$ value as shown in the hatched area. The broken line in Fig. 4 
shows estimation on the assumption of continuous change of the carrier concentration for PbTe. This gradient PbTe has an ideal FGM structure and exhibits a higher $Z$ than the segmented $\mathrm{PbTe}$ over a wide temperature range. It is estimated that the average $\mathrm{Z}$ of the ideal FGM should improve by $50 \%$ in comparison with a monolithic $\mathrm{PbTe}$, and maximum thermoelectric efficiency $\eta$ at $\mathrm{T}_{\mathrm{h}}=950 \mathrm{~K}$ should extend to $19 \%$.

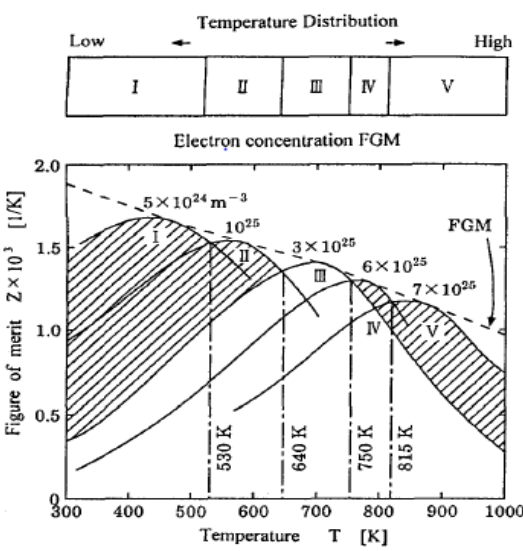

Fig. 4. Temperature dependence of $\mathrm{Z}$ for $\mathrm{n}$-type $\mathrm{PbTe}$ as parameters of camer concentration[4]

This concept can be applied not only to the carrier concentration gradient but also to joining different materials, such as $\mathrm{Bi}_{2} \mathrm{Te}_{3}-\mathrm{PbTe}-\mathrm{SiGe}$ etc., corresponding to the temperature gradient.

\section{CONCLUSIONS}

1) FGM belongs to novel material category and offers new capabilities to use it at large scale. FGM technology has the potential to drastically redefine the methods used for developing lighter, stronger, and high performance structures with unique and nontraditional properties. FGM-composites often lead to a reduction in weight and costs, and are more environmental friendly. For these reasons the popularity of these composites is increasing in engineering world. However, engineering breakthroughs will be required to develop the technology in long-term goals. Technologies expect the FGM kind of advanced materials with revolutionary new capabilities such as in power systems, electronic devices, sensors, science and medical diagnostic instruments.

2) Functionally graded materials are perspective materials for modern optoelectronic devices, such as low threshold current edge lasers (GRINSCH) and tuneable photo detectors. Graded layers can also be used as buffers in heteroepitaxy of nitrides. Development and characterization of FGM is a real challenge and needs thorough analysis. The methodology of measuring layers with graded changing properties is difficult. Another problem is the interrelation between different material properties. It is different to change one parameter like energetic band-gap, without influencing the others like refractive index.

3) The thermoelectric materials with FGM structure show a higher performance than monolithic materials. FGM joining is also a useful technique for setting an electrode in order to relax the thermal stress and suppress the inter diffusion. However, it is difficult to improve the performance by controlling crystal size. On the other hand, exact measurement of thermal conductivity at a high temperature is another problem for accurate designing of an FGM.

4) A temperature sensor results if one of the junctions is maintained at a well-known temperature. The thermocouple can also be used as an actuator. The advantages of TE energy conversion is that moving mechanical parts are avoided which enables high system reliability, quiet operation, and it is usually environmentally friendly. It has been found that the tellurium compounds $\mathrm{Bi}_{2} \mathrm{Te}_{3}$ and $\mathrm{Sb}_{2} \mathrm{Te}_{3}$ having $\mathrm{ZT}>2$ are well established TE materials for futuristic uses.

\section{REFERENCES}

[1] K. M. Gupta, Materials Science, Metallurgy and Engineering Materials, Umesh Publications, New Delhi, 2012

[2] G. Udupal, S. S. Rao, and K. V. Gangadharan, "Future applications of Carbon Nanotube reinforced Functionally Graded Composite Materials," in Proc. IEEE-International Conference on Advances in Engineering, Science and Management (ICAESM -2012), March 3031, 2012.

[3] A. S. Maiolo, M. N. Amado, and J. S. Gonzalez et al., "Development and characterization of poly (vinyl alcohol) based hydrogels for potential use as an articular cartilage replacement," Mater. Sci. Eng. C, 2012, vol. 32, no. 6, pp. 1490-1495.

[4] G. Abedi, A. Sotoudeh, M. Soleymani et al., "A collagenpoly(vinyl alcohol) nanofiber scaffold for cartilage repair," J. Biomater. Sci., Polym. Ed., 2011, vol. 22, no. 18, pp. 2445-2455.

[5] M. Wosko, B. Paszkiewicz, T. Piasecki, A. Szyszka, R. Paszkiewicz, and M. Tlaczala, "Application of functionally graded materials in optoelectronic devices," optical Application, vol. 35, no. 3, 2005.

[6] I. Shiota and I. A. Nishida, "Development of FGM thermoelectric materials in Japan-the state of the art," 16th International Conference on Thermoelectrics, IEEE, pp. 364-370, 1997.

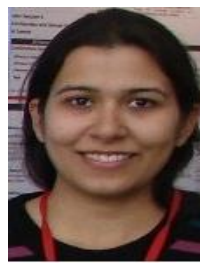

Isha Bharti is from New Delhi, India. She completed her Masters (M.Tech.) in Nanoscience and Technology with specialization in Nanobiotechnology from Delhi Technologica University in 2011. Earlier, she did her under graduation (B.Tech.) in Biotechnology from Maharshi Dayanand University, Rohtak, Haryana. Her major field of study is synthesis and fabrication of Nanobiotechnological materials, luminescent materials for novel biomedical applications and diagnostic therapies, etc. 